\title{
Labyrinthe
}

$5 \mid 2000$

Numéro 5

\section{Comprendre la violence}

\section{(2) OpenEdition}

1 Journals

Édition électronique

URL : http://journals.openedition.org/labyrinthe/286

DOI : 10.4000/labyrinthe.286

ISSN : 1950-6031

Éditeur

Hermann

Édition imprimée

Date de publication : 15 janvier 2000

Pagination : 129-132

Référence électronique

"Comprendre la violence », Labyrinthe [En ligne], 5 | 2000, mis en ligne le 17 février 2005, consulté le

22 septembre 2020. URL : http://journals.openedition.org/labyrinthe/286 ; DOI : https://doi.org/

10.4000 /labyrinthe. 286

Ce document a été généré automatiquement le 22 septembre 2020.

Propriété intellectuelle 


\section{Comprendre la violence}

Depuis le début de cette année universitaire, Labyrinthe organise chaque mois un « séminaire d'ouverture» destiné aux étudiants de première année du Centre de Formation des Journalistes (CFJ). Nous vous proposons ici un rapide compte rendu de la première de ces interventions (2 novembre 1999), établi à partir de notes d'auditeurs. Elle est due à Xavier Crettiez, maître de conférences à Paris II et auteur de l'article intitulé « Violence symbolique d'État contre violence terroriste basque » (Labyrinthe, $\mathrm{n}^{\circ} 2$, p. 59-75).

1 Un lieu commun voudrait que nos sociétés industrialisées connaissent une « violence grandissante ». Rien n'est moins sûr. Il y a incontestablement plus de violence en ce sens qu'elle est plus visible - continuellement mise en spectacle par les médias - et surtout que son seuil de tolérance s'est considérablement élevé. Ce qui ne veut pas dire, bien au contraire, qu'elle soit plus présente dans les comportements - statistiquement, elle a décru. On a affaire à une disproportion considérable entre sa perception et sa " réalité effective " : c'est d'une certaine manière parce qu'il y a moins de violence que l'on en parle plus...

2 Cela étant dit, le concept de violence est particulièrement difficile à appréhender. Une définition classique et restrictive de ce terme fait appel à son étymologie latine : vis (force) et latus (participe passé de fero, qui signifie porter). C'est alors le fait de porter une force sur quelqu'un ou quelque chose. Cette définition est celle de la violence physique, la plus durement ressentie et la plus visible. Mais les choses se compliquent dès lors que l'on cherche à appréhender les notions de violences douces, selon l'expression de Michel Foucault (schématiquement : une violence qui ne s'exerce plus sous la forme des supplices infligés par le pouvoir étatique, mais par une surveillance accrue) ou encore de violence symbolique, concept utilisé par Pierre Bourdieu pour qualifier une violence qui tire sa légitimité d'un accord implicite des victimes.

3 Mais ce ne sont pas ces approches de la violence qui intéressent ici Xavier Crettiez. Son objet est la violence physique et les formes de son exercice. Il en distingue trois : instrumentale, passionnelle, identitaire.

4 L'exercice de la violence instrumentale est dirigé vers un objectif précis ; c'est une violence rationnelle, stratégique et toujours proportionnée au but à atteindre. La " coercition d'État » vise, paradoxalement, à assurer la non-violence dans la société dont il est l'ex-pression : garantir la sécurité interne (les illégalismes) ou externe (la guerre). 
Cette violence instrumentale ne concerne pas seulement le pouvoir d'État ; elle peut être également un mode d'expression contestataire, choisi par les organisations dites terroristes, qui recourent à la lutte armée pour faire reconnaitre leur souveraineté ou faire triompher leurs idéaux, contre l'État « colonial » (ETA, IRA, FLNC), «bourgeois » (AD, BR, RAF) ou « impur » (GIA). Xavier Crettiez a particulièrement insisté, autour de l'exemple corse qu'il connait bien, sur la violence comme mode d'insertion dans la vie politique. Dans une société où le jeu politique est fondé sur un système de type clanique, la violence apparaît comme un moyen rationnel d'accéder à la reconnaissance politique. Dans le même ordre d'idées, des organisations parfaitement légales peuvent recourir à une violence non pas "symbolique " mais plutôt " symbolisée " comme moyen d'expression et de pression. On pense notamment aux paysans sous la $\mathrm{V}^{\mathrm{e}}$ République ou encore aux étudiants. De toutes les façons cette catégorie de violence implique une forte exigence d'organisation, voire d'institutionnalisa-tion, afin d'assurer que son exercice ne se détourne pas des buts qui lui avaient été assignés. Il en va tout autrement de la violence dite passionnelle.

Cette dernière peut être définie comme un «acting out » destructeur, provoqué par une décharge d'agressivité et correspondant à une suspension du calcul rationnel. Ses caractères distinctifs - qui relèvent davantage de la psychologie que de la sociologie sont au nombre de quatre : elle est impulsive et imprévisible tant en ce qui concerne son déclenchement que son apaisement ; elle est discontinue dans son exercice ; elle est peu durable ; elle est souvent disproportionnée par rapport à ses objectifs.

6 Trois écoles interprétatives se sont penchées sur cette forme de violence. Les thèses de type " éthologiste " se concentrent sur la question du caractère inné ou acquis des dispositions individuelles à la violence colérique. Par le passé, ces thèses furent développées notamment par les théoriciens évolutionnistes des foules, tel Gustave Le Bon. Aujourd'hui, les sciences sociales ont abandonné cette question, jugée insoluble. En revanche, elle intéresse des neuro-biologistes comme Boris Cyrulnik. Le deuxième ensemble de théories repose sur le concept de frustration relative qui résulte du décalage entre attentes et réalisations. Plus le décalage est important, plus la frustration est élevée et la violence colérique. Le schéma descriptif de ce comportement est le suivant : frustration ${ }^{\circledR}$ colère ${ }^{\circledR}$ identification d'une cible ${ }^{\circledR}$ infliction d'un dommage ${ }^{\circledR}$ réduction de la colère. Le troisième et dernier grand ensemble de modèles explicatifs fait appel aux mécanismes culturels d'intériorisation et d'apprentissage de la violence. Aux comportements colériques individuels préexiste une culture spécifique fournissant des modèles de référence agressifs qui valorisent la violence comme une source de reconnaissance et de gratification. Si les sociétés archaïques en donnent de nombreux exemples, certaines « subcultures » occidentales accordent aussi une valeur centrale à la force physique associée au culte des valeurs viriles.

7 S'apparentant aux deux formes de violence précédentes, la violence identitaire se présente comme un moyen de construire et d'af-firmer l'identité d'un groupe et au contraire à dénier celle d'un autre groupe. Ainsi est-ce un véritable langage, un outil de définition communautaire. Parce qu'elle est une exhibition de puissance, la violence peut servir à renforcer la solidarité d'un groupe violent, à affirmer son unité en dépassant les particularités individuelles. L'image du "cagoulé » confère à l'indépendantiste corse une visibilité politique qui contribue à construire l'identité du FLNC. Le cas le plus frappant est sans doute celui du Rwanda où la violence a fortement contribué à la fabrication d'identités conflictuelles. La distinction entre Hutus et Tutsis 
ne repose sur aucune base réelle, ni raciale ni culturelle - le Rwanda est un pays linguistiquement et culturellement homogène. C'est l'administration coloniale (belge et allemande) qui, à des fins politiques, a inventé et construit cette distinction au moyen des outils de la science moderne (science morphologique, procédés d'identification...). En 1995, cette opposition importée aboutit à un conflit entre des races qui n'ont de réalité que dans les représentations de soi et de l'autre. Paradoxalement, la violence génocidaire fait exister ce qu'elle prétendait exprimer : une totale opposition identitaire - si l'autre est assassiné de façon barbare, c'est nécessairement parce qu'il est un barbare.

8 La violence apparaît alors comme le moyen du déni identitaire. Les atrocités commises dans les camps nazis relèvent bien moins de la violence colérique ou de la violence instrumentale que du désir de refuser à une communauté l'appartenance au genre humain. Un exemple significatif : le chien du commandant SS de Treblinka avait été appelé " homme ", alors que les juifs mangeaient par terre, dans des gamelles. La perversité extrême de la violence concentrationnaire consiste à faire exister ce qui la motive : faire que ses victimes, traitées comme des bêtes, en adoptent le comportement, et se perçoivent elles-mêmes comme des bêtes. Les témoignages de Primo Lévi et de David Rousset rendent compte de ce terrible processus d'intériorisation. 